\title{
Comparison of Various Image Compression Transforms
}

\author{
S.V. Phakade ${ }^{1}$, Rushikesh Patil ${ }^{2}$, Aishwarya Patil $^{3}$, Rahael More $^{4}$ \\ ${ }^{I}$ Associate Professor at Department of Electronics and Telecommunication, PVPIT, Budhgaon, Maharashtra. \\ ${ }^{2,3,4}$ B.E. Students at Department of Electronics and Telecommunication, PVPIT, Budhgaon, Maharashtra, India. \\ Email: rushipatil241297@gmail.com ${ }^{2}$
}

\begin{abstract}
This paper presents image compression using various Transforms. Transforms like DCT, DST, Slant, Walsh and Haar are applied on colour image. Full transform, column transform and Row transforms are three methods used for images compression. In each method Image is Transformed and reconstructed using Inverse Transform. Root mean Square (RMSE) and Peak Signal to noise ratio between Original and compressed image is Calculated. From Implementation of these Transforms it has been observed that DCT, DST, slant transforms are gives better result in terms of RMSE, PSNR and Visual Quality of image. Haar and Walsh Transform gives acceptable result with good Compression Ratio. Aim of compression using Transform is to compress Image without high losses and Achieve good visual Quality after Compression.
\end{abstract}

Keywords-Image compression, lossy compression, full transform, column transform, row Transform, matlab.

\section{INTRODUCTION}

Use of Internet, Multimedia and their Application is increased. These applications Required Images and videos more than Text data. Images and video represent more visual information as compared to Text. Image and video used for many purposes in communication, education, entertainment and many other areas. In that case, problems of storage, processing, time consumption and transmission is high. Easy way to overcome these problems is compression of Data. When Image compression is come into picture, storage space reduction and maintaining visual quality of image is also important. Lossless and lossy are two main technique of image compression. In Lossless compression, original image and compressed have similarities as there is no loss of information. In case of lossy compression, loss of information is high with high compression. lossy compression techniques provide higher compression ratio as compared to lossless compression techniques. Transform based Image compression is lossy compression technique.

Discrete cosine Transform (DCT) and Discrete Sine Transform (DST) are most commonly and widely used as compared to slant, Haar and Walsh for image compression. These transforms effectively compress an image with maintaining good image quality.

\section{TRANSFORM BASED COMPRESSION}

Transform coding is linear process. It is used to convert spatial image pixel values to transforms coefficient values. No of coefficient produced is equal to number of pixels transformed. Transform based technique uses

linear process of mapping pixel values onto set of coefficients. It is lossy compression technique there is loss of information. JPEG (Joint Photographic Experts Group) is most commonly used for lossy compression.

\subsection{Discrete Cosine Transform}

Discrete Cosine transform (DCT) transforms real data points into its real spectrum. Computational efficiency is high as compared to other transforms. DCT convert image into frequency component. Dct developed by N. Ahmed, T. Natarajan, K.R. Rao in 1974. Dct used for image and video processing, pattern recognition and filtering.

\subsection{Discrete Sine Transform}

Discrete Sine Transform (DST) is Fourier related transform similar to Discrete Fourier Transform and Discrete Cosine Transform with some change in Properties. Dct is fast, real, symmetric and orthogonal Transform.

\subsection{Slant Transform}

Slant is unitary transform especially designed for image coding. Slant transform has high energy compaction, fast computational algorithm, variable size transformation. ${ }^{[14]}$

\subsection{Haar Transform}

Haar transform introduced by Hungarian Mathematician Alfred Haar in 1910. Haar transform matrix contains ones and zeros. hence it requires no multiplication and less number of addition. Haar transform is fast, compact and orthonormal wavelet transform. ${ }^{[15]}$

\subsection{Walsh Transform}

Walsh transform also known as Walsh-Hadamard transform, hadamard-rademacher-walsh transform. It is ultra-fast, orthogonal, symmetric, involutive transform. Walsh functions are rectangular and square waveforms with values of +1 or -1 . Walsh transform used for data encryption and signal processing.

\section{PURPOSED TECHNIQUE}

In this compression technique each Transform is applied on image to obtain transformed image. These transforms are applied in three different ways: full transform, column transform and row transform. Let 
International Journal of Research in Advent Technology, Vol.7, No.4, April 2019

E-ISSN: 2321-9637

Available online at $w w w . i j r a t . o r g$

'I' denotes an image, ' $M$ ' denotes transformation matrix, ' $\mathrm{T}$ ' indicates transformed image. Then,

Full Transform $[\mathrm{T}]=[\mathrm{M}] *[\mathrm{I}] *\left[\mathrm{M}^{\prime}\right]$

Where $\mathrm{M}^{\prime}$ is Transpose of $\mathrm{M}$.

For Column Transform $[\mathrm{T}]=[\mathrm{M}] *[\mathrm{I}]$

For Row Transform [T] = [ I] *[ M']

In each of these three cases, low energy coefficients are eliminated from transformed image content. Then image is reconstructed by applying inverse transform on it. In column transform, number of coefficients is reduced by eliminating some rows of transformed image. In row transformation, it is done by eliminating some columns of transformed image. In Full transform some rows as well as some columns are eliminated such that number of coefficients reduced is equal as that of column or row transform.

Image is then reconstructed by applying inverse transform on that image which contains reduced number of coefficients than original image. Root mean square error (RMSE), Peak signal to noise ratio (PSNR) and compression ratio is calculated in each case till acceptable image quality is obtained. Average of all these ratios are used for performance analysis. ${ }^{[5]}$

\section{RESULTS}

15 sample colour images are used for experimentation. Experiments are performed in MATLAB 9.4.0. (R2018a) on laptop with core i5 processor and 8 GB RAM. Test images are shown in figure 1.
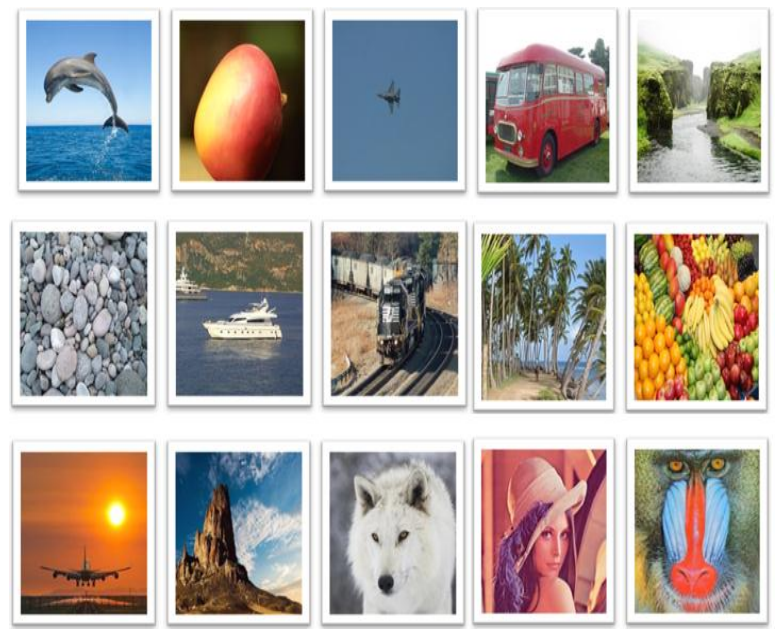

Figure 1. Set of fifteen test images of different classes used for experiment namely (from left to right and top to bottom) Dolphin, Mango, Jet, Bus, River, Stones, Cruise, Train, Beach, Fruits, Airplane, Mountain, Wolf, Lenna, Mandrill.

For Comparison images are resized into 256x256x3, $320 \times 480 \times 3,640 \times 640 \times 3$ and resize applied to each set of five images from set of fifteen test images, figure 1 . For DCT, comparison of three cases i.e. Ratios in full transform, column transform and row transform are shown in Table 1 to 3 .
Table 1. Average of Compression ratio (CR), RMSE and PSNR for DCT full transform.

\begin{tabular}{|l|l|l|l|}
\hline \multirow{2}{*}{$\begin{array}{l}\text { Image } \\
\text { Resize }\end{array}$} & \multicolumn{3}{|l|}{ FULL TRANSFORM } \\
\cline { 2 - 5 } & $\begin{array}{l}\mid \\
\text { AVG } \\
\end{array}$ & $\begin{array}{l}\text { AVG } \\
\text { RMSE }\end{array}$ & \multicolumn{1}{|l|}{$\begin{array}{l}\text { AVG } \\
\text { PSNR }\end{array}$} \\
\hline & 11.48792 & $2.74 \mathrm{E}-14$ & 735.68782 \\
\hline $256 \times 256 \times 3$ & 4.12756 & $2.58 \mathrm{E}-14$ & 736.8998 \\
\hline & \multicolumn{3}{|c|}{} \\
\hline $320 \times 480 \times 3$ & 2.49884 & $2.71 \mathrm{E}-14$ & 735.7956 \\
\hline
\end{tabular}

Table 2. Average of CR, RMSE and PSNR for DCT Column Transform.

\begin{tabular}{|c|c|c|c|}
\hline \multirow{3}{*}{$\begin{array}{l}\text { IMAGE } \\
\text { RESIZE }\end{array}$} & \multicolumn{3}{|c|}{ COLUMN TRANSFORM } \\
\hline & AVG & AVG & $\mathrm{AVG}$ \\
\hline & $\mathrm{CR}$ & RMSE & PSNR \\
\hline $256 \times 256 \times 3$ & 11.48792 & $1.92 \mathrm{E}-14$ & 742.7376 \\
\hline $320 \times 480 \times 3$ & 4.12756 & $1.81 \mathrm{E}-14$ & 743.8231 \\
\hline $640 \times 640 \times 3$ & 2.49884 & $1.89 \mathrm{E}-14$ & 742.9853 \\
\hline
\end{tabular}

Table 3. Average of CR, RMSE and PSNR for DCT Row Transform.

\begin{tabular}{|c|c|c|c|}
\hline \multirow{3}{*}{$\begin{array}{l}\text { IMAGE } \\
\text { RESIZE }\end{array}$} & \multicolumn{3}{|c|}{ ROW TRANSFORM } \\
\hline & $\overline{A V G}$ & $\mathrm{AVG}$ & $\mathrm{AVG}$ \\
\hline & CR & RMSE & PSNR \\
\hline $256 \times 256 \times 3$ & 11.48792 & $1.98 \mathrm{E}-14$ & 742.1447 \\
\hline $320 \times 480 \times 3$ & 4.12756 & $1.84 \mathrm{E}-14$ & 743.4081 \\
\hline $640 \times 640 \times 3$ & 2.49884 & $1.95 \mathrm{E}-14$ & 742.4406 \\
\hline
\end{tabular}

It can be observed that Column and Row transform gives better RMSE ratio as compared to Full transform. In full transform acceptable image Quality is maintained.

Table 4 represents Summary of Average of CR, RMSE and PSNR for full Transform applied on first five images with resize 256x256x3.

Table 4. Summary of average CR, RMSE and PSNR for various Full Transforms applied on images with resize $256 \times 256 \times 3$. 
International Journal of Research in Advent Technology, Vol.7, No.4, April 2019

E-ISSN: 2321-9637

Available online at www.ijrat.org

\begin{tabular}{|l|l|l|l|}
\hline $\begin{array}{l}\text { Name } \\
\text { of } \\
\text { Transform }\end{array}$ & AVG CR & $\begin{array}{l}\text { AVG } \\
\text { RMSE }\end{array}$ & $\begin{array}{l}\text { AVG } \\
\text { PSNR }\end{array}$ \\
\hline DCT & 11.48792 & $2.74 \mathrm{E}-14$ & 735.68782 \\
\hline DST & 11.48792 & $3.37 \mathrm{E}-14$ & 731.65794 \\
\hline SLANT & 11.48792 & $2.18 \mathrm{E}-14$ & 740.30528 \\
\hline HAAR & 11.45341 & $1.89 \mathrm{E}-01$ & 159.25828 \\
\hline WALSH & 11.37106 & $2.24 \mathrm{E}+00$ & 98.09032 \\
\hline
\end{tabular}

Table 5 represents Summary of CR, RMSE and PSNR for full transforms applied on next five images from test image set with resize $320 \times 480 \times 3$.

Table 5. Summary of average of CR, RMSE and PSNR for various full Transforms applied on image with resize $320 \times 480 \times 3$.

\begin{tabular}{|l|l|l|l|}
\hline $\begin{array}{l}\text { Name } \\
\text { of } \\
\text { Transform }\end{array}$ & $\begin{array}{l}\text { AVG } \\
\text { CR }\end{array}$ & $\begin{array}{l}\text { AVG } \\
\text { RMSE }\end{array}$ & $\begin{array}{l}\text { AVG } \\
\text { PSNR }\end{array}$ \\
\hline & & & \\
\hline DCT & 4.1275 & $2.76 \mathrm{E}-14$ & 736.68998 \\
\hline DST & 4.1275 & $2.98 \mathrm{E}-14$ & 734.80536 \\
\hline SLANT & 4.1275 & $2.01 \mathrm{E}-14$ & 741.63936 \\
\hline HAAR & 4.1246 & $2.22 \mathrm{E}-01$ & 142.71238 \\
\hline WALSH & 7.2722 & $4.06 \mathrm{E}+00$ & 82.93774 \\
\hline
\end{tabular}

Table 6 gives Summary of Average of CR, RMSE and PSNR for Full transforms applied on last images from test image set with resize 640x640x3.

Table 6. Summary of Average of CR, RMSE and PSNR for various full Transforms applied on images with resize $640 \times 640 \times 3$.

\begin{tabular}{|l|l|l|l|}
\hline $\begin{array}{l}\text { Name } \\
\text { of } \\
\text { Transform }\end{array}$ & $\begin{array}{l}\text { AVG } \\
\text { CR }\end{array}$ & $\begin{array}{l}\text { AVG } \\
\text { RMSE }\end{array}$ & $\begin{array}{l}\text { AVG } \\
\text { PSNR }\end{array}$ \\
\hline & & & \\
\hline DCT & 2.49884 & $2.17 \mathrm{E}-14$ & 735.79564 \\
\hline DST & 2.49884 & $3.17 \mathrm{E}-14$ & 732.65856 \\
\hline SLANT & 2.49884 & $2.14 \mathrm{E}-14$ & 740.5359 \\
\hline HAAR & 2.4997 & $1.58 \mathrm{E}-01$ & 155.65786 \\
\hline WALSH & 3.69902 & $1.93 \mathrm{E}+00$ & 98.58318 \\
\hline
\end{tabular}

From fifteen test images 'Mandrill' image used for comparison. It contains different colour combination and edges. Compressed image obtained by applying full transform, row transform, column transform is shown below with corresponding CR, RMSE value of each image.
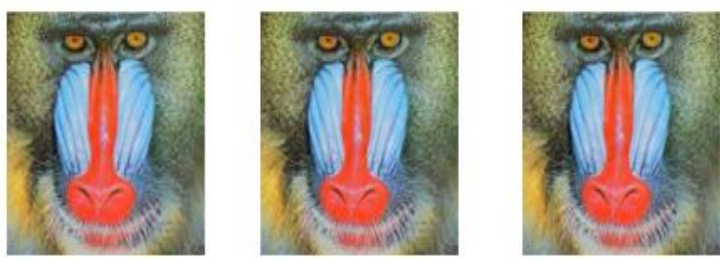

$\mathrm{RMSE}=2.7252 \mathrm{e}-14 \mathrm{RMSE}=1.9104 \mathrm{e}-14 \quad \mathrm{RMSE}=1.9569 \mathrm{e}-14$

$$
\begin{array}{lll}
\text { PSNR }=735.4985 & \text { PSNR }=742.6029 & \text { PSNR }=742.1218 \\
\text { CR }=9.6754 & \text { CR }=9.6754 & \text { CR }=9.6754
\end{array}
$$

Figure 2. Compressed Image by applying DCT Full, Column and Row Transform.

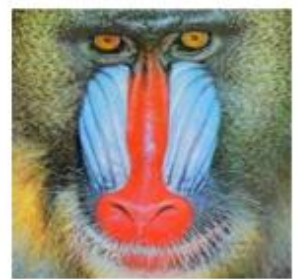

Full DST Transform

$$
\begin{aligned}
& \text { RMSE }=3.1516 \mathrm{e}-14 \\
& \text { PSNR }=732.5909
\end{aligned}
$$$$
\mathrm{CR}=9.6754
$$

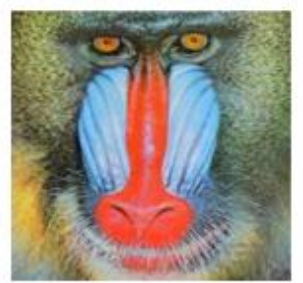

Full Slant Transform

RMSE $=2.1350 \mathrm{e}-14$

PSNR $=740.3798$

$\mathrm{CR}=9.6754$
Figure 3. Compressed Image by applying Full DST and Slant transform.

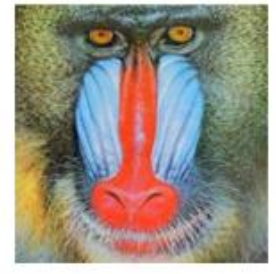

Full Haar Transform

$$
\mathrm{RMSE}=0.0847
$$$$
\text { PSNR }=160.2001
$$$$
\mathrm{CR}=9.6760
$$

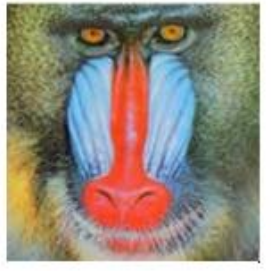

Full Walsh Transform

RMSE $=3.2602$

$\mathrm{PSNR}=87.1893$

$\mathrm{CR}=11.7176$
Figure 4. Compressed Image by applying Full Haar and Walsh Transform.

\section{CONCLUSION}

Performance analysis is done by comparing Root Mean Square Error (RMSE), Peak Signal to Noise Ratio (PSNR) and Compression Ratio (CR). Obtained Result is in form of $\mathrm{E}^{-14}$ which indicates smaller values. RMSE Ratio should be small as it Proves the Image Quality after Compression by Comparing Original image and Compressed Image. Result proves that RMSE values obtained shows that Column and Row transform gives better result as compared to Full transform. For better RMSE and PSNR column and Row Transform can be used. It saves half number of computation and maintain good image quality. For 
DCT, DST and Slant Transforms obtained RMSE ratio is in form of E-14 which is smaller than Haar and Walsh. PSNR values of these transforms are higher than Haar and Walsh. Haar Transform gives Result in form of $\mathrm{E}^{-01}$ which is higher than $\mathrm{E}^{-14}$. Walsh Transform gives higher value of RMSE than Haar Transform. From all six Transforms performed DCT, DST and Slant Transform give better result in terms of RMSE, PSNR and Reconstructed Image quality than other Transforms. Haar and Walsh transforms also give good result with acceptable image quality with moderate RMSE value.

\section{REFERENCES}

[1] Jayaraman S., Digital Image Processing.

[2] A. Jain, Fundamentals of Digital Image Processing, Prentice-Hall, 1989.

[3] Ahmed, N., Natarajan T., Rao K. R.: Discrete cosine transform. In: IEEE Transactions on Computers, Vol. 23, 90-93, 1974

[4] H.B.Kekre, Tanuja Sarode, Prachi Natu, "Efficient Image Compression Technique Using Full, Column and Row Transforms on Colour Image", International Journal of Advances in Engineering and Technology, Vol.6, Issue 1, March 2013, pp. 88-100.

[5] H.B.Kekre, Tanuja Sarode, Sudeep Thepade, "Inception of hybrid wavelet Transform using Two orthogonal Transforms and It's use for Image compression", IJCSIS, vol 9, no. 6, 2011.

[6] H.B.Kekre, Tanuja Sarode, Sudeep Thepade, "Color Image Compression using Hybrid Wavelet Transform with Haar as Base Transform", International Journal of Scientific and Research Publications, Volume 4, Issue 6, June 20141 ISSN 2250-3153 www.ijsrp.org

[7] H. B. Kekre, Tanuja Sarode, Prachi Natu, "Image Compression Using Column, Row and Full Wavelet Transforms of Walsh, Cosine, Haar, Kekre, Slant and Sine and Their Comparison with Corresponding Orthogonal Transforms", International Journal of Engineering Research and development (IJERD), Vol. 6, Issue 4, March 2013, pp.102-113.

[8] Dipti Bhatnagar, Sumit Budhiraja, "Image Compression using DCT based Compressive Sensing and Vector Quantization", IJCA, Vol50 (20), pp. 34-38, July 2012

[9] Er. Abhishek Kaushik, Er. Deepti Nain "Image Compression Algorithms Using Dct" Int. Journal of Engineering Research and Applications www.ijera.com ISSN: 2248-9622, Vol. 4, Issue 4(Version 1), April 2014, pp.357-364

[10] Sonal Chawla, Meenakshi Beri, Ritu Mudgil, "Image Compression Techniques: A Review",International Journal of Computer Science and Mobile Computing, Vol.3 Issue.8, August- 2014, pg. 291-296
[11]T. Vimal Prakash Singh, "Matlab Implementation of Baseline JPEG Image Compression Using Hardware Optimized Discrete Cosine Transform", International Journal of Engineering Science Invention ISSN (Online): 2319 - 6734, Volume 3 Issue 8 August 2014 PP.47-53.

[12] Analysis of Image Compression Methods Based on Transform and Fractal Coding - a Thesis by Archana Deshlahra.

[13] W. K. Pratt, W.H.Cheng and L. R. Welch, "Slant Transform Image Coding", IEEE Trans. commn. Vol. Comm. 22, pp. 1075-1093, Aug. 1974.

[14] R.S. Stanković and B.J. Falkowski. "The Haar wavelet transform: its status and achievements". Computers and Electrical Engineering, Vol.29, No.1, pp.25-44, January 2003.

[15] Prabhakar.Telagarapu, V.Jagan Naveen, A.Lakshmi Prasanthi, G.Vijaya Santhi, "Image compression using DCT and wavelet transformation", IJSPIPPR, vol 4, issue 3, pp. 6174, Sept 2011.

[16] Image compression and DCT by ken Cabeen and Geter gent. 